\title{
A survey of Galileo plasma wave instrument observations of Jovian whistler-mode chorus
}

\author{
J. D. Menietti ${ }^{1}$, R. B. Horne ${ }^{2}$, D. A. Gurnett ${ }^{1}$, G. B. Hospodarsky ${ }^{1}$, C. W. Piker $^{1}$, and J. B. Groene ${ }^{1}$ \\ ${ }^{1}$ University of Iowa, Dept. Physics and Astronomy, Iowa City, IA 52242-1479, USA \\ ${ }^{2}$ British Antarctic Survey, Madingley Road, Cambridge CB3 OET, UK
}

Received: 6 December 2007 - Revised: 23 April 2008 - Accepted: 16 May 2008 - Published: 1 July 2008

\begin{abstract}
A survey of plasma wave observations at Jupiter obtained by the plasma wave instrument on board the Galileo spacecraft is presented. The observations indicate that chorus emissions are observed commonly in the Jovian magnetosphere near the magnetic equator in the approximate radial range $6<r<10 R_{J}$. The survey includes almost all local times but not equally sampled in radial distance due to the spacecraft trajectory. The data suggest that chorus emissions are somewhat more intense on the dayside, but this may be a result of insufficient nightside observations. The orbit of Galileo is also restricted to $\pm 3^{\circ}$ of the Jovigraphic equator, but the tilt of the magnetic field permits coverage of a range of magnetic latitudes of $-13^{\circ}<\lambda_{\mathrm{mag}}<+13^{\circ}$. The similarities of chorus emissions to terrestrial observations are a good reason to speculate that Jovian chorus emission may play a significant role in the stochastic acceleration of electrons in the radial range 6-10 $R_{J}$ as recent studies indicate. These electrons may then be transported inward by radial diffusion where they are additionally accelerated to form the synchrotron radiation belt source.
\end{abstract}

Keywords. Magnetospheric physics (Energetic particles, trapped; Planetary magnetospheres) - Space plasma physics (Wave-particle interactions)

\section{Introduction and background}

\subsection{Terrestrial chorus emissions}

It has been previously shown (e.g. Tsurutani and Smith, 1974; Anderson and Maeda, 1977) that the injection of terrestrial substorm electrons leads to the excitation of intense whistler mode chorus emissions in the vicinity of the geomagnetic equator outside of the plasmasphere. These waves,

Correspondence to: J. D. Menietti

(john-menietti@uiowa.edu) in turn, can accelerate the electrons in the Earth's outer radiation belt to relativistic $(\mathrm{MeV})$ energies as suggested by Horne and Thorne (1998) (see also Summers et al., 2002; Meredith et al., 2003a).

At Earth, intense, diffuse whistler mode hiss at frequencies typically less than a few kilohertz is observed within the plasmasphere. The source of plasmaspheric hiss is currently a subject of investigation, but recently Bortnik et al. (2008) have suggested that plasmaspheric hiss may originate from whistler mode chorus waves. Natural emissions of discrete, whistler-mode chorus at Earth consist of electromagnetic waves in the frequency range from a few hundred hertz to several $\mathrm{kHz}$ observed in the lower density region outside the plasmapause. These emissions are most often characterized as narrow-banded at $f<f_{c e} / 2$ and/or $f>f_{c e} / 2$ with an emission gap near $f_{c e} / 2$, where $f_{c e}$ is the electron cyclotron frequency. It is generally believed that chorus is generated by a nonlinear process based on the electron cyclotron resonance of whistler-mode waves with energetic electrons, taking place close to the geomagnetic equatorial plane (Omura et al., 1991; Nunn et al., 1997; LeDocq et al., 1998; Trakhtengerts, 1999; Santolik et al., 2003, 2004). Storm-time chorus is especially important for the physics of the Earth's magnetosphere since it can significantly influence the distribution functions of the energetic electrons in the outer radiation belt (e.g. Meredith et al., 2003a; Horne and Thorne, 2003).

\subsection{Jovian chorus}

Chorus observations at Jupiter were first detected by Voyager 1 using the 16 channel spectrum analyzer data (cf. review by Gurnett and Scarf, 1983). The chorus emissions were confined to a single frequency channel and were observed close to the magnetic equator. No extensive survey of chorus emission at Jupiter has been possible until the Galileo mission.

Published by Copernicus Publications on behalf of the European Geosciences Union. 
The radiation belts at Jupiter are remotely observed as strong sources of synchrotron emission (cf. Berge and Gulkis, 1976; Carr et al., 1983; Bolton et al., 2001) The emission has a peak power between $80 \mathrm{MHz}$ and $300 \mathrm{GHz}$ near $1.4 R_{J}$ where electrons with energies up to $50 \mathrm{MeV}$ can be found. These energies are much more energetic than those typically observed in the Earth's radiation belt, and there is a question regarding the source of electron energization at Jupiter as at Earth. While radial diffusion and the interchange instability along with conservation of the adiabatic invariants can explain energization of electrons from $1 \mathrm{MeV}$ to over $10 \mathrm{MeV}$, there is still the question of what energizes the electrons to $1 \mathrm{MeV}$ in the middle magnetosphere (cf. Hill et al., 1983; Brice and McDonough, 1973; Thorne et al., 1997; Bolton et al., 1997).

Centrifugal force from the rapid rotation of the planet drives flux interchange instabilities whereby outward transport of cold dense plasma is replaced by inward transport of higher energy particles that develop a temperature anisotropy and excite whistler mode waves (Bolton et al., 1997; Thorne et al., 1997). This concept is discussed in some detail by Hill et al. (1983). See also Fig. 12.12 of Thorne (1983) which depicts the flow of centrifugally driven plasma in the Jovian magnetosphere. The interchange instability results from radial transport of plasma in a rotationally-dominated magnetosphere. Ideally this would be manifested by alternating longitudinal sectors of cooler, denser plasma moving outward and hotter, more tenuous plasma moving inward (cf. Pontius et al., 1986; Hill et al., 1983). Thorne et al. (1997) have indicated that the most unstable interchange motions should be confined to the inner portions of the Io torus $(6<L<8)$. As we show in the present study, the intensity of whistler mode waves is a maximum in a region between $6-10 R_{J}$, where flux interchange instabilities are observed. Thus the region beyond Io $\left(\sim 6 R_{J}\right)$, and near the outer regions of the torus $\left(\gtrsim 8 R_{J}\right)$ where the density falls off, provides a natural and plentiful source of energy to drive the waves unstable. The Io torus is composed of ions and neutral atoms including sodium, sulfur, and oxygen whose source is primarily the volcanic eruptions on the moon. The torus extends from about $5 R_{J}$ to beyond $8 R_{J}$, and completely envelopes the orbit of Io at about $6 R_{J}$, but the ionic density has a centroid near the magnetic equator or centrifugal symmetry surface. The maximum ionic densities near the magnetic equator can be greater than $3000 \mathrm{~cm}^{-3}$ (cf. Bagenal, 1994). Recent studies of Horne et al. (2008) have shown the effectiveness of stochastic acceleration by chorus at Jupiter. These authors used chorus data observed by the Galileo plasma wave instrument for a specific case to model electron energy and pitch angle diffusion as well as electron acceleration. The assumed power levels were based on plasma wave observations at $10 R_{J}$ and subsequently scaled according to the average radial profile of the wave intensity measured by Galileo (averaged over all longitudes) within 10 degrees of the magnetic equator. Horne et al. (2008) find that in the ab- sence of radial transport, the electron flux between $1-6 \mathrm{MeV}$ increases by more than an order of magnitude after a period of 30 days (see their Fig. 4a). The results clearly show the effectiveness of wave-particle interactions in the overall particle dynamics at Jupiter. These studies also indicate the need for improved statistical studies of chorus emissions at Jupiter to provide improved parameters for existing models of chorus-stimulated electron acceleration. This need was a motivation to conduct the present study.

\subsection{Whistler mode emission}

The whistler mode instability can be driven by a pitch-angle anisotropy which is commonly observed near the Earth's magnetic equator. Assuming the high density approximation in the cold plasma approximation, the resonance velocity for field-aligned electrons, as described in Gurnett and Bhattacharjee (2005), is obtained from the dispersion relation and the cyclotron non-relativistic resonance condition for parallel wave numbers as

$v_{\|_{\operatorname{Res}}}=\frac{-c\left(\omega_{c e}-\omega\right)^{3 / 2}}{\left(\omega_{p} \omega^{1 / 2}\right)}$,

and we can thus define resonance energy $W_{\|_{\text {Res }}}$ and a characteristic energy $W_{c}$ that identifies whistler-mode electron resonance interactions, which is strongly dependent on the ratio of plasma frequency to cyclotron frequency $\omega_{p} / \omega_{c e}$,

$$
\begin{aligned}
& W_{\|_{\mathrm{Res}}}=W_{c}\left(1-\frac{\omega}{\omega_{c e}}\right)^{3}\left(\frac{\omega_{c e}}{\omega}\right) \\
& W_{c}=\frac{1}{2} m c^{2}\left(\frac{\omega_{c e}}{\omega_{p}}\right)^{2},
\end{aligned}
$$

where $m$ is the electron mass and $c$ is the speed of light. For the region outside the terrestrial plasmasphere (and also the Jovian Io torus) $W_{c}$ can be several $\mathrm{keV}$ and higher, and we can expect whistler mode wave particle interactions, electron scattering and acceleration as suggested for Earth by Horne and Thorne (1998) and Meredith et al. (2003a), for instance. Note that relativistic corrections have been studied by Horne and Thorne (1998), and these are important at high energies, $E>100 \mathrm{keV}$.

The whistler mode growth rate is dependent on an anisotropy factor, which for a bi-temperature Maxwellian distribution can be written as

$A=T_{\perp} / T_{\|}-1$,

where $T_{\perp}$ and $T_{\|}$are the electron temperature perpendicular and parallel to the magnetic field. Temperature anisotropies are observed in the particle distributions near the terrestrial magnetic equator (cf. Horne et al., 2003), and these distributions are unstable to the growth of whistler mode emission. 
In this paper we conduct a survey of whistler mode chorus plasma wave emissions observed by the plasma wave instrument (PWS) on board the Galileo spacecraft. These emissions may have substantial impact on the energization of the Jovian electron population as these particles are transported from the middle magnetosphere to the inner radiation belts.

\section{Instrumentation}

The Galileo plasma wave instrument (PWS) provides excellent spectral frequency resolution over a range extending from a few $\mathrm{Hz}$ to almost $6 \mathrm{MHz}$ (electric) and $\sim 160 \mathrm{kHz}$ (magnetic). Since Galileo is an orbiter, in contrast to past flyby missions, it provides unique local time information and continuous mapping of the equatorial magnetosphere in the radial distance range to over $140 R_{J}$. The inclination of the orbits was about 3 degrees, for the Galileo orbit insertion and orbit G1. Beginning with orbit $\mathrm{G} 2$, the inclination was altered to near zero. The tilt of the magnetic axis of $\sim 10^{\circ}$ yields a total range of magnetic latitude of $-10^{\circ}$ to $+10^{\circ}$ for the bulk of the mission. The orbits vary in ellipticity, perijove and apojove, but the range of sampled distances is from about $5 R_{J}$ to over $140 R_{J}$.

The plasma wave receiver on board Galileo consists of 4 different swept-frequency receivers that cover the frequency range from $5.6 \mathrm{~Hz}$ to $5.6 \mathrm{MHz}$ for electric fields and $5.6 \mathrm{~Hz}$ to $160 \mathrm{kHz}$ for magnetic fields. We will concentrate in this study on the electric fields obtained by the mediumfrequency receiver, which covers the frequency range from $42.1 \mathrm{~Hz}$ to $160.8 \mathrm{kHz}$. A single electric dipole antenna with a tip-to-tip length of $6.6 \mathrm{~m}$ is connected to each electric receiver. A complete set of electric field measurements is obtained every $18.67 \mathrm{~s}$ with a frequency resolution of about $10 \%$ (cf. Gurnett et al., 1992).

\section{Observations}

Chorus observations were obtained by PWS from each perijove pass of Galileo. Frequency-vs-time spectrograms of the radio emissions were analyzed for narrow-banded emissions below the electron cyclotron frequency and above the more diffuse whistler mode emissions at lower frequency. In Fig. 1 we show one of the better examples of chorus emissions observed on orbit G7, near the Ganymede flyby. The plot is a frequency-vs-time spectrogram over the frequency range from $100 \mathrm{~Hz}$ to $100 \mathrm{kHz}$ with electric (Fig. 1a) and magnetic field intensity (Fig. 1b) color-coded as indicated in the bar at the right of each figure. The magenta line in Fig. 1a indicates the location of the upper hybrid emission in the approximate range $40 \mathrm{kHz}<f<70 \mathrm{kHz}$. The magnetic spectrogram (Fig. 1b) shows a number of interference lines indicated with arrows. Chorus emission is seen in the frequency range from a few hundred $\mathrm{Hz}$ up to, but not exceeding, approximately $f_{c e} / 2$, where $f_{c e}$ is indicated by the white line. We are somewhat limited by the frequency resolution of the low-rate data plotted, but no high resolution data is available at this time. The electromagnetic whistler mode chorus emission is seen below the electron cyclotron frequency, $f_{c e}$, in the approximate frequency range $400 \mathrm{~Hz}<f<8 \mathrm{kHz}$. The emission shows a repeating narrow-banded structure with maximum frequency occurring near the magnetic equator crossings of Jupiter. The banded emission above $f_{c e}$ is electrostatic electron cyclotron harmonic $(\mathrm{ECH})$ emission that is commonly observed near the magnetic equator at Earth (cf. Kurth et al., 1979; Gough et al., 1979), and has recently been studied at Saturn (Menietti et al., 2008). We note that chorus emission reaches maximum frequency away from the magnetic equator, and there is an absence of emission close to the equator. The time between crossings of the magnetic equator in Fig. 1 varies from $\sim 5.5-6 \mathrm{~h}$ due to the motion of the spacecraft as well as the thickness of the plasma sheet. The chorus emission electric field spectral densities (peaks near $\left.10^{-10} V^{2} /\left(\mathrm{m}^{2}-\mathrm{Hz}\right)\right)$ are comparable but somewhat smaller than those observed at Earth by CRRES, Polar, and Cluster (cf. Meredith et al., 2001; Lauben et al., 1998; Santolik et al., 2003; Horne et al., 2005). The decrease in frequency of the chorus as a function of magnetic latitude is a common feature of the emission at Jupiter and is observed sometimes in terrestrial chorus emission as well, but the magnitude of the frequency change at Jupiter is much larger. Since the frequency of chorus is dependent on the resonance velocity as well as $f_{p}$, the implication is that the electron density at Jupiter has a strong dependence on magnetic latitude. We know this is true in the torus region due to centrifugal forces. It is possible that higher frequency chorus emission does not propagate as close to the magnetic field direction as the low frequency emission. It is also possible that Landau damping as a function of latitude may be a contributing factor (cf. Bortnik et al., 2007). We point out that the chorus emission observed at Saturn also displays a similar, strong frequency dependence on magnetic latitude (Hospodarsky et al., 2008).

In Fig. 2 we present a much smaller time and frequency interval from Fig. 1a. The white line still indicates $f_{c e}$. Note the upper and lower bands of chorus emission (indicated by arrows) with a band of omission near $f_{c e} / 2$. In Fig. 3 we present the average spectral density versus frequency in the range $2 \mathrm{kHz}$ to $7 \mathrm{kHz}$ for the one-minute time interval 17:00 to 17:01 UT. The electric and magnetic spectral densities are displayed in Fig. 3a and b, respectively. We note in these plots the chorus intensity gap near $4.5 \mathrm{kHz}$, at about $f_{c e} / 2$. The lines on the horizontal axis of Fig. 3a, b indicate the location of $f_{c e} / 2$ based on the average value of the magnetic field during the one minute time interval. This upper and lower band morphology of chorus is commonly observed for terrestrial chorus near the magnetic equator and may indicate similar processes occurring at Earth and Jupiter. Both upper and lower bands occurred for portions of the chorus emission on segments of approximately 10 of the 30 orbital passes examined. 

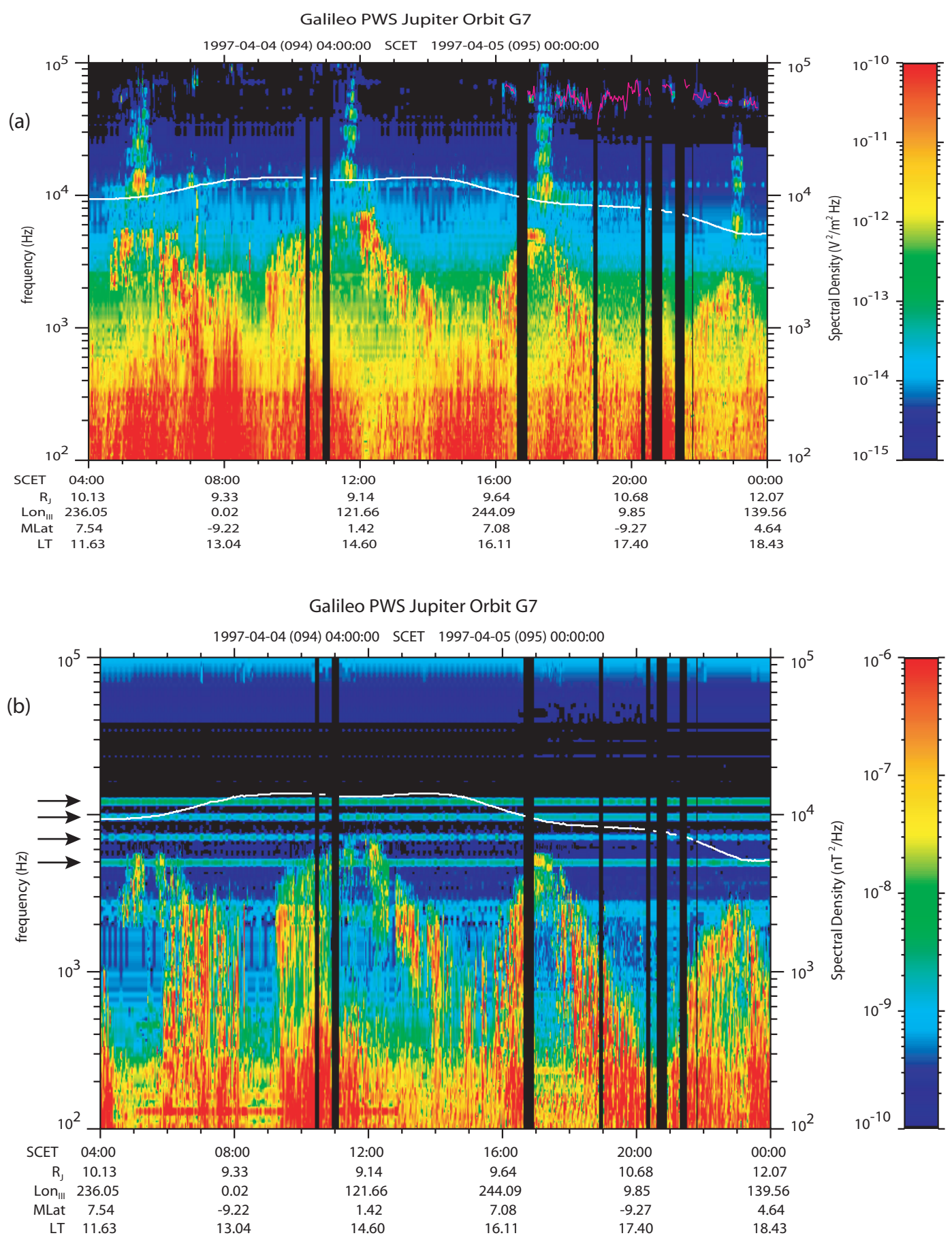

Fig. 1. An example of chorus emissions observed on orbit G7. The plot is a frequency-vs-time spectrogram over the frequency range from $100 \mathrm{~Hz}$ to $100 \mathrm{kHz}$ with electric (a) and magnetic field intensity (b) color-coded as indicated in the bar at the right of each panel. The magenta line in panel (a) indicates upper hybrid emission.

The data for the survey were examined in the form of frequency-vs-time spectrograms such as Figs. 1 and 2, obtained for each perijove pass. A database of time and frequency range was compiled for each example of chorus emission observed. Chorus was observed on 30 of the orbits examined, essentially each time the satellite and wave instrument were properly functioning in the radial distance $5 R_{J}<r<12 R_{J}$. For the purposes of this study chorus was defined to be relatively narrow-banded emission at frequencies above the more continuous and broad-banded (hiss-like) 


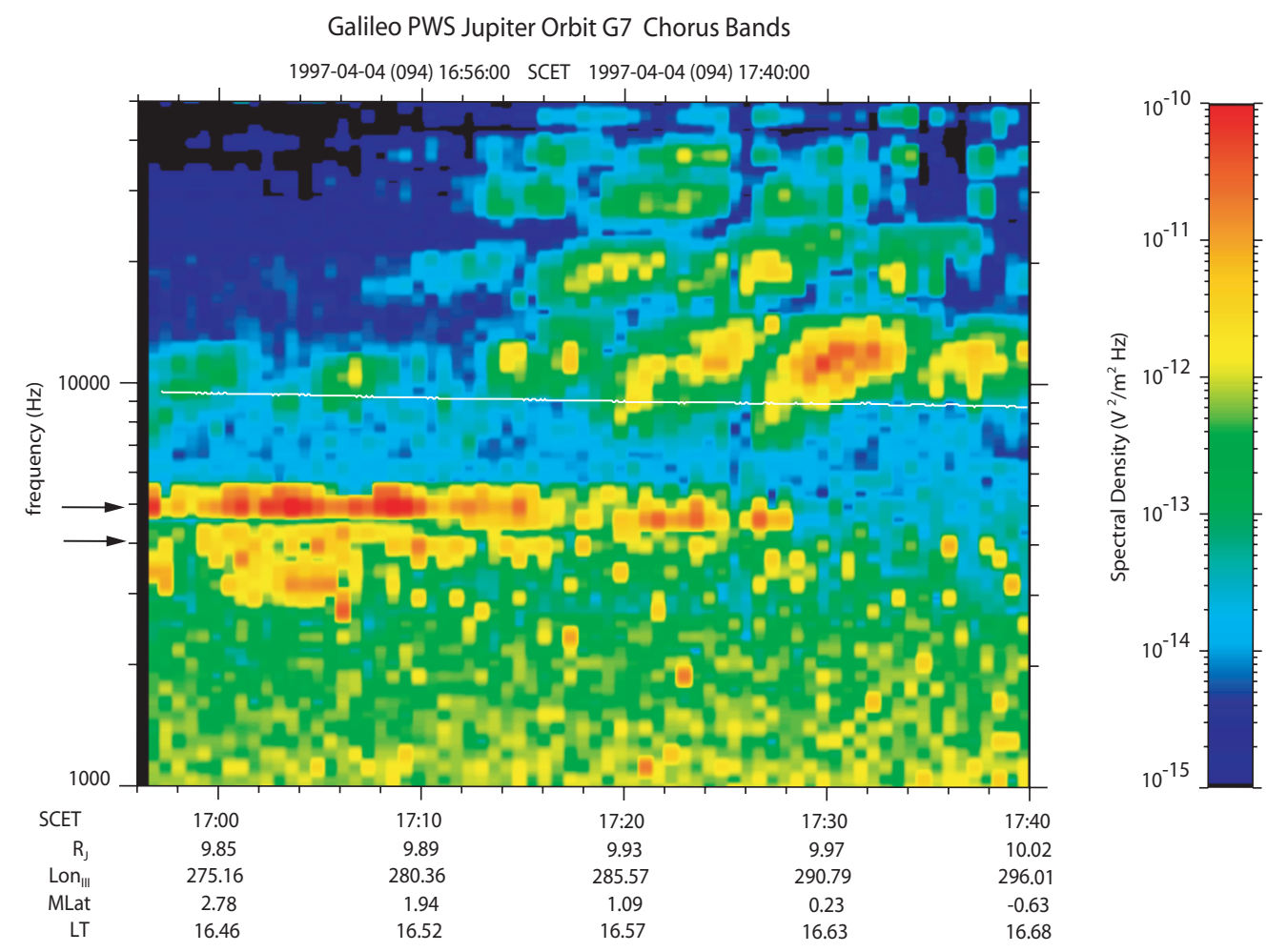

Fig. 2. A smaller time and frequency interval from Fig. 1a. The white line still indicates $f_{c e}$. Note the upper and lower bands of chorus emission (indicated by arrows) with a band of omission near $f_{c e} / 2$.

emission almost always observed at $f \lesssim 300 \mathrm{~Hz}$. In Fig. 1 the chorus emission most probably extends to frequencies below $300 \mathrm{~Hz}$, but we typically chose a conservative approach and set the lower frequency limit at a frequency where mixing of hiss and chorus emissions was apparent. For Fig. 1 we chose a lower frequency limit of $400 \mathrm{~Hz}$, and no data for $f<300 \mathrm{~Hz}$ was ever included in the calculation of chorus power levels. So the reported frequency-integrated powers for the chorus emission represent a probable lower limit to the actual intensities. Chorus was also defined to be emission below $f_{c e}$. For all observations of chorus, the plasma frequency, $f_{p}$, was greater than $f_{c e}$ as confirmed when upper hybrid emissions were observed.

Both electric and magnetic intensity levels are received by the PWS, but the magnetic receivers suffered increasing degradation after orbit C9, in the second half of 1997, which resulted in extremely high background levels for frequencies less than $2.8 \mathrm{kHz}$ and enhanced levels of interference. Attempting to account for this by reducing the frequency range of integration for the chorus power levels for the magnetic receiver would have resulted in an incomplete survey of the data and it was impossible to accurately compare the results to the electric power levels, so it was decided to include only the electric receiver power levels for this survey.

In Fig. 4 we show the results of the complete survey in a plot of portions of the trajectory during which chorus emis- sion was observed. The color code indicates the frequencyintegrated power spectral density in units of $(\mathrm{V} / \mathrm{m})^{2}$. The plot is in solar equatorial coordinates with the sun in the $+\mathrm{X}$ direction and dawn in the $+\mathrm{Y}$ direction. It appears there is more chorus power on the dayside, but there is a lack of orbital coverage on the nightside in the range $r<15 R_{J}$, so no definitive conclusions can be drawn. Chorus is observed for $r>10 R_{J}$ at almost all local times except near dawn. There is also a data gap due to lack of orbital coverage near $20 \mathrm{~h}$ of local time (LT). It is difficult to compare Fig. 4 with terrestrial global maps of chorus emission. At Earth chorus emission occurs with maximum amplitude on the dawnside and at periods of increased auroral activity (cf. Plate 4 of Meredith et al., 2001). At Earth you expect plasma injections and drift due to convection through dawn, but at Jupiter convective injection is less likely as co-rotation tends to dominate. The lack of Jovian chorus for $r>10 R_{J}$ at dawn may be due to flux interchange being less effective in this region. The Galileo mission represents an incomplete sampling of local time, and the effects of magnetic storms and solar wind are not considered. Solar wind conditions at Jupiter during the Galileo emission are not directly measured, and observations of auroral images (related to magnetospheric activity) are not continuous.

In Figs. 5, 6, and 7 we plot the power levels as a function of radial distance, magnetic latitude, and local time, 

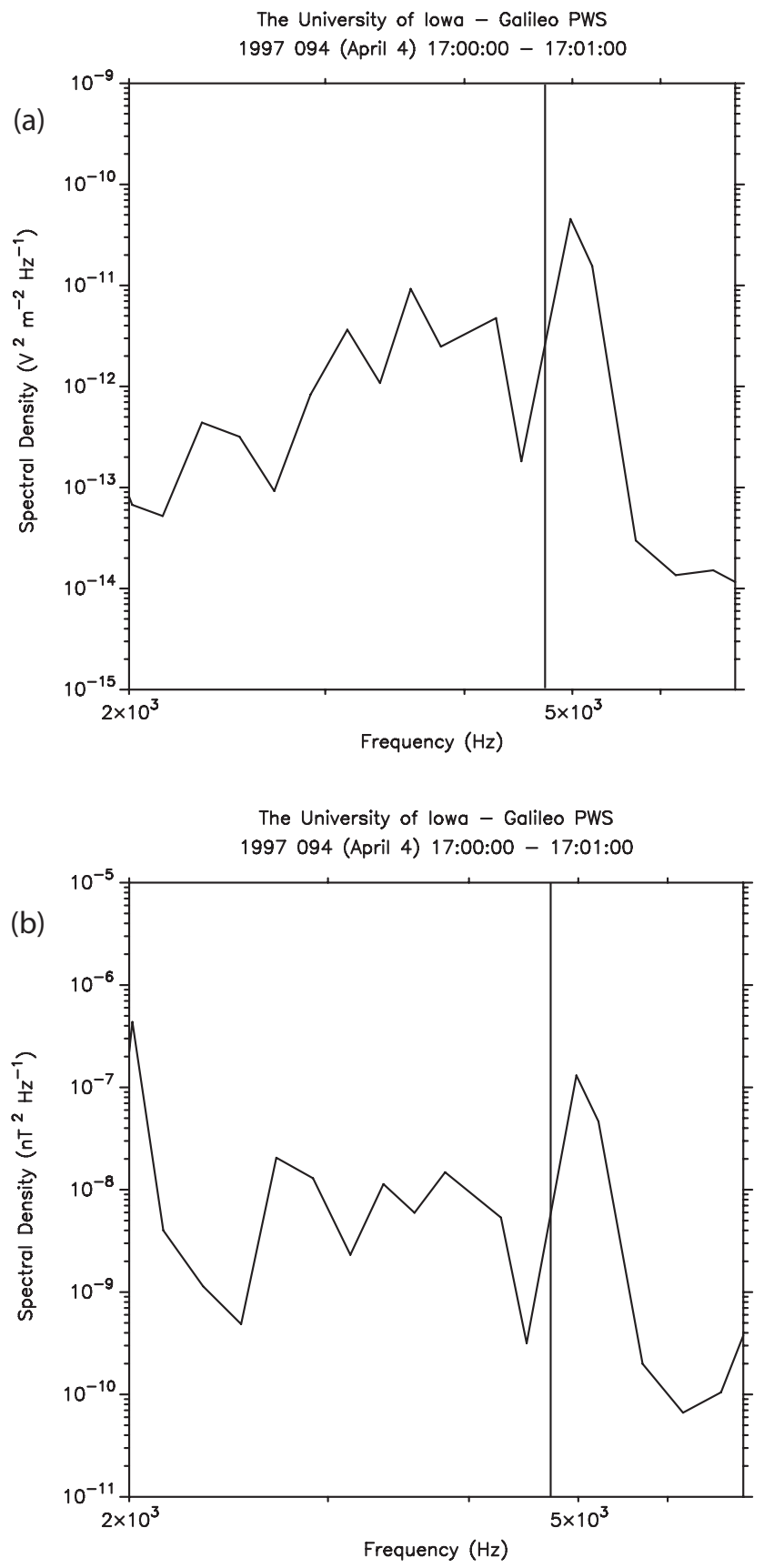

Fig. 3. The average spectral density versus frequency in the range $2 \mathrm{kHz}$ to $7 \mathrm{kHz}$ for the one-minute time interval 17:00 to 17:01 UT. The electric and magnetic spectral densities are displayed in (a) and (b), respectively. The vertical lines of panels (a), (b) indicate the location of $f_{c e} / 2$ based on the average value of the magnetic field during the one minute time interval.

respectively. The top panel of each figure shows the number of frequency sweeps within each bin. There are 50 bins for each range of parameter. The power levels are obtained by integrating the spectral densities over the frequency range of chorus observations. The values plotted represent the aver-

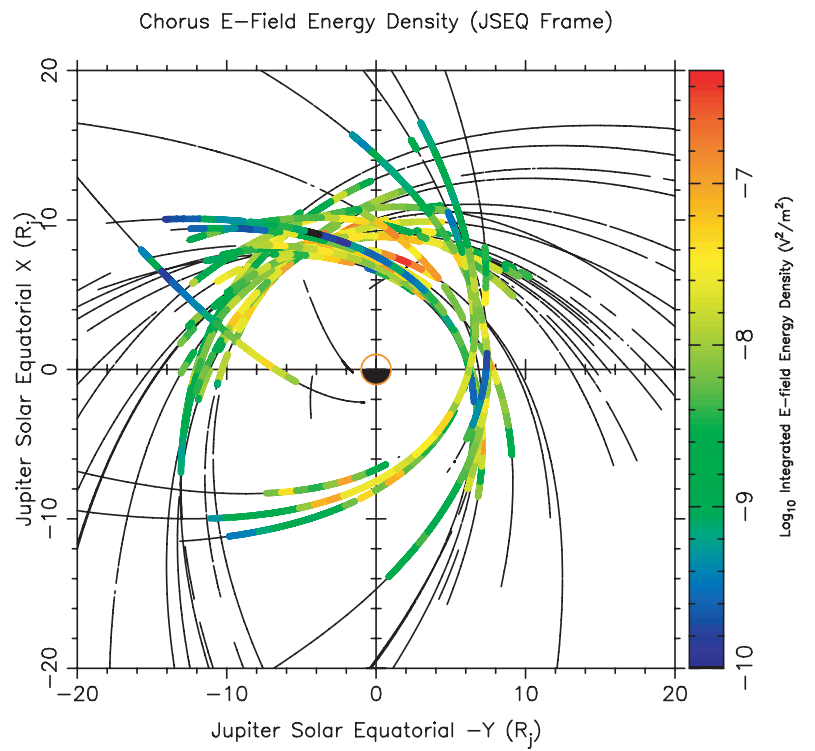

Fig. 4. The results of the complete survey in a plot of portions of the trajectory during which chorus emission was observed. The color code indicates the frequency-integrated power spectral density in units of $(\mathrm{V} / \mathrm{m})^{2}$. The plot is in solar equatorial coordinates with the sun in the $+\mathrm{X}$ direction and dawn in the $+\mathrm{Y}$ direction.

age power within each bin. The power levels show a general peak $\left(P>\sim 10^{-8} \mathrm{~V}^{2} / \mathrm{m}^{2}\right)$ that lies in the approximate range of $6<r<10 R_{J}$. In local time the power levels are more distributed, but there is a distinct decrease in power centered near a LT of $20 \mathrm{~h}$ due at least in part to orbit constraints and a lack of data for $r<10 R_{J}$ at this LT. Power levels show minor peaks between $11 \mathrm{~h}<\mathrm{LT}<14 \mathrm{~h}$ with other peaks near midnight and near 3 to $4 \mathrm{~h} \mathrm{LT}$. In magnetic latitude the power levels show a broad peak on each side of the magnetic equator, but then fall off at both $-10^{\circ}$ and $+10^{\circ}$. There is a clear minimum of power near the magnetic equator which increases by about a factor of 4 near $\pm 3^{\circ}$. This increase in power away from the magnetic equator is consistent with nonlinear resonant currents as discussed by Katoh and Omura (2006, 2007). Falloff of power at higher latitudes may be explained by Landau damping as discussed by Bortnik et al. (2007). The fall-off at high latitude is real, for there are fewer, but nevertheless a significant number of sweeps and many orbits sampled at the higher latitudes as seen in the upper panel of Fig. 6. Only for $\lambda_{\mathrm{mag}}>10^{\circ}$ are the data limited by few orbits as mentioned earlier. It is interesting that the power decreases faster for positive latitudes than for negative. This is also true if the data is plotted versus centrifugal latitude (not shown). The minimum near $\lambda_{\text {mag }}=0$ is not inconsistent with recent studies. Katoh and Omura (2007), for instance, show that chorus power grows away from the magnetic equator due to nonlinear trapping of resonant electrons. 

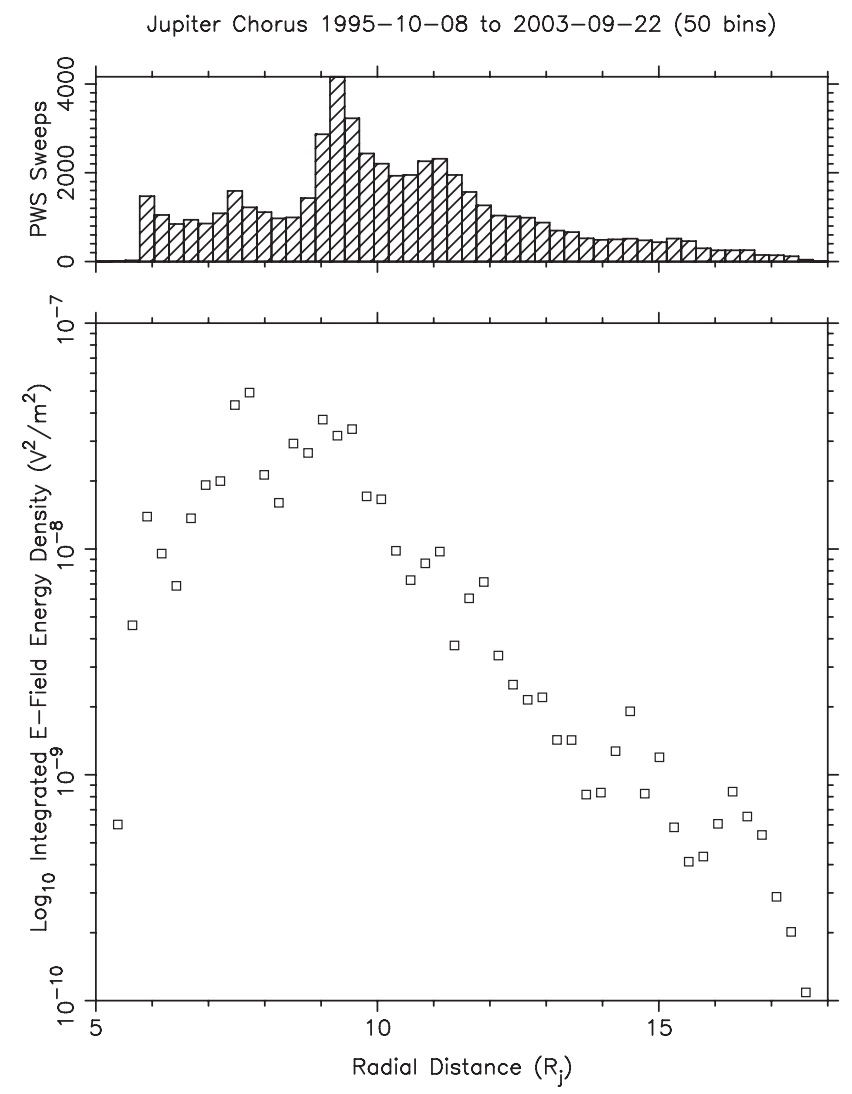

Fig. 5. Integrated chorus power as a function of radial distance (lower panel). In this and in the following figures the top panel shows the number of frequency sweeps within each bin. There are 50 bins for each range of parameter.

\section{Discussion}

The observations of chorus emission at Jupiter show many similarities to those at Earth, but also some distinctive and interesting differences. Chorus is observed near the magnetic equator at Jupiter and at Earth. At Jupiter the power reaches a local minimum at the equator and peaks a few degrees away. A similar effect is seen in terrestrial chorus emission observed by the Polar spacecraft (K. Sigsbee, private communication, 2008), but the effect at Jupiter is quite distinctive. As mentioned above, this observation may be explained by the recent non-linear simulations of Katoh and Omura (2006, 2007). The spectral densities of the emission at Jupiter do not appear to be as high as those observed at Earth, by perhaps an order of magnitude and sometimes more. The chorus emissions at Earth are primarily seen during enhanced magnetic activity outside the plasmapause in the approximate range $3<L<7$ (e.g. Meredith et al., 2003b) where the critical ratio $f_{p} / f_{c e}$ rapidly decreases making energy diffusion by whistler mode cyclotron resonance with ambient electrons more effective (Summers et al., 1998; Horne et al., 2003). The role of the plasmapause at Jupiter is replaced by the Io torus.
Jupiter Chorus $1995-10-08$ to $2003-09-22$ (50 bins)
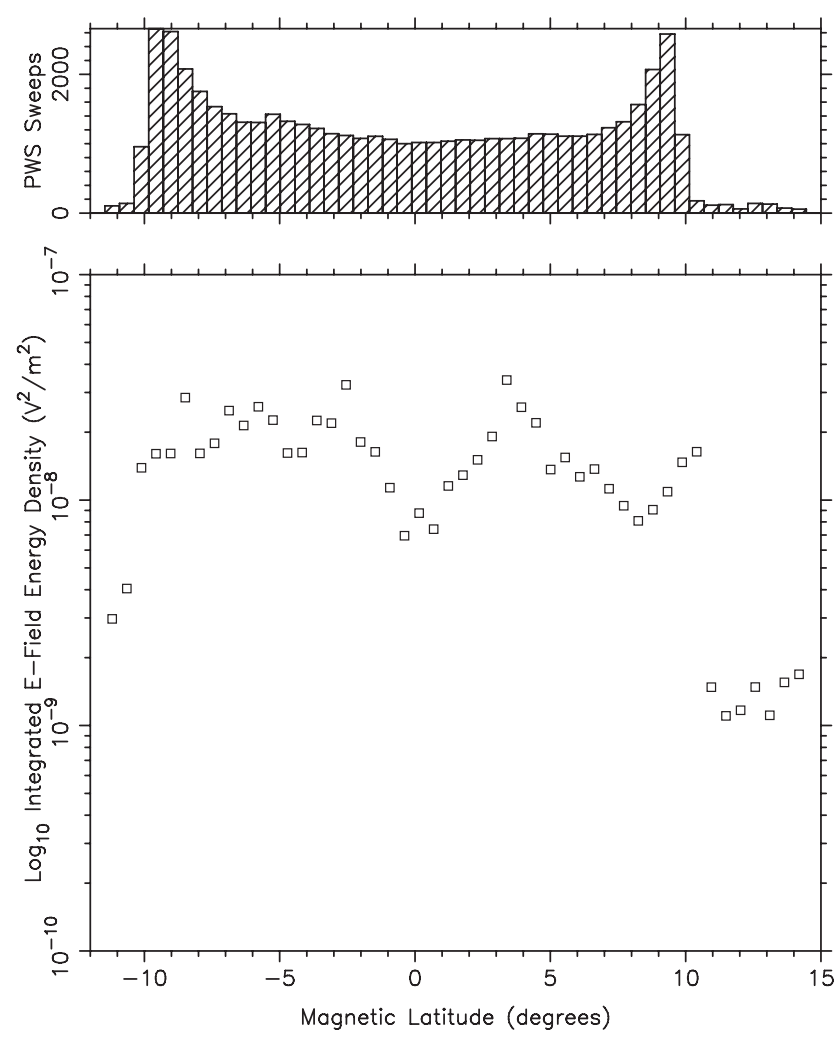

Fig. 6. Integrated chorus power as a function of magnetic latitude.

Chorus emission levels are seen to be highest in the range $6<r<10 R_{J}$ where plasma densities decrease radially after a peak near $r=5 R_{J}$ within the torus. This region at Jupiter largely overlaps with the region where the flux interchange instability is important. Conservation of the first and second adiabatic invariants within distant, warm, density-depleted magnetic flux tubes that are radially transported inward develop electron distributions with a temperature anisotropy that is unstable to the growth of whistler mode emission. While at Earth it is believed that plasma injections provide a seed population that lead to enhanced chorus generation and acceleration of electrons (Summers et al., 2002), the interchange instability may play a similar role at Jupiter in the range $6<r<10 R_{J}$.

Horne et al. (2008) have performed pitch angle and energy diffusion as well as Fokker-Planck calculations demonstrating that gyro-resonant acceleration of electrons at Jupiter is most effective outside the orbit of Io. They show that the electron flux at a few $\mathrm{MeV}$ increases by an order of magnitude after a period of 30 days. For these calculations the chorus power spectrum was assumed to be a Gaussian in frequency within $\pm 10^{\circ}$ magnetic latitude with no latitudinal dependence. The assumed wave power levels were based on Galileo plasma wave spectral density observations at $10 R_{J}$, scaled according to the average radial profile, and averaged 
Jupiter Chorus $1995-10-08$ to $2003-09-22$ (50 bins)
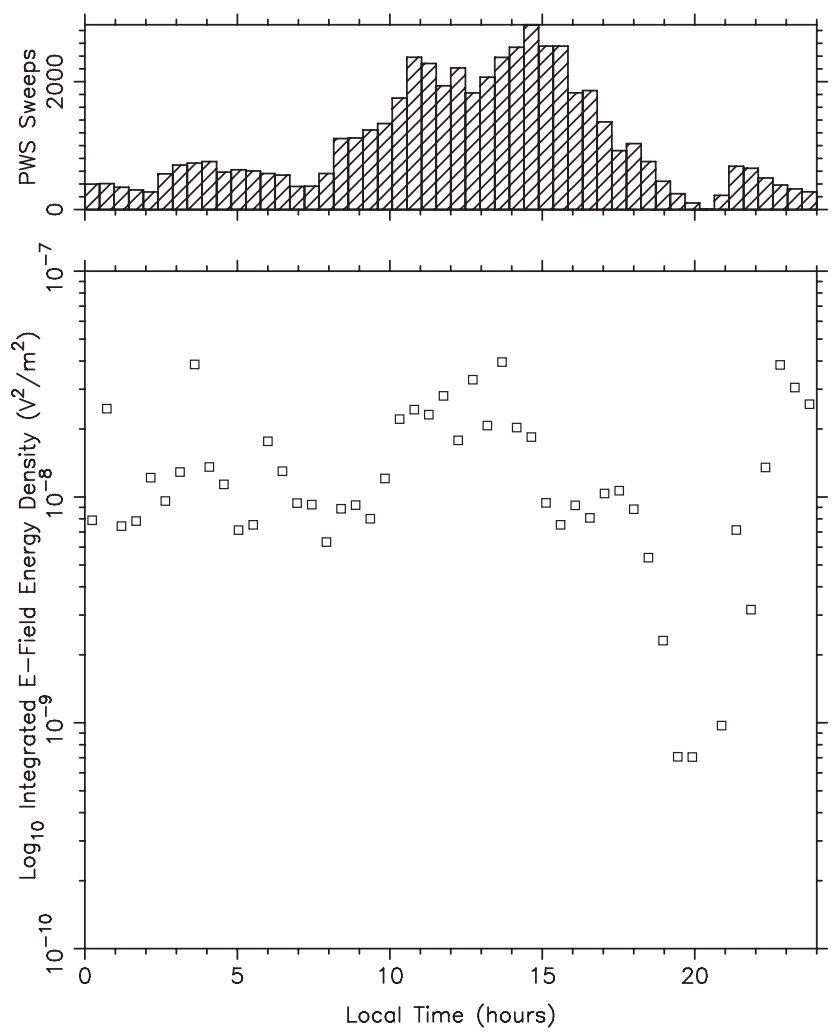

Fig. 7. Integrated chorus power as a function of local time.

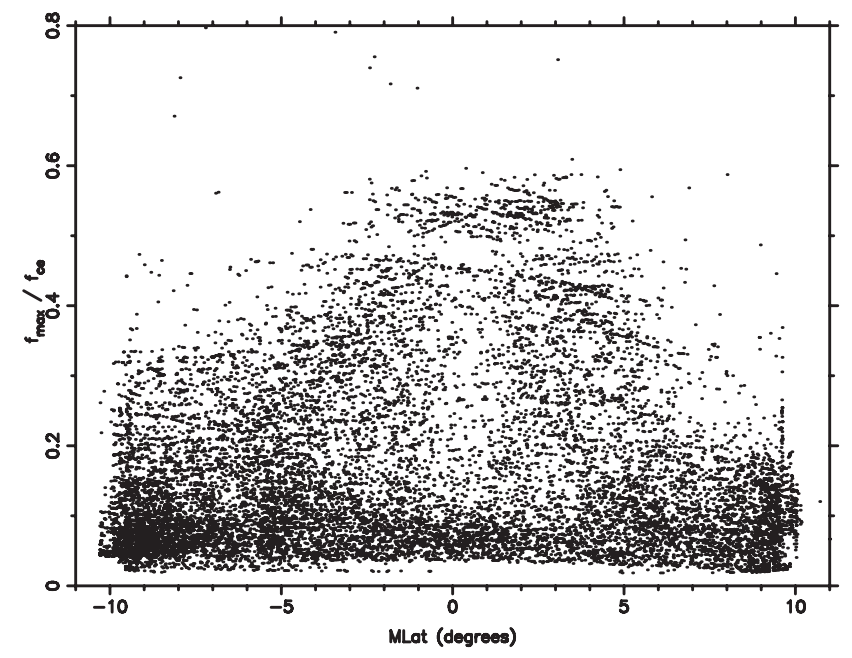

Fig. 8. A plot of the upper cutoff of the chorus frequency normalized to $f_{c e}$ versus magnetic latitude. Each point represents a value obtained from an algorithm that analyzed each frequency sweep of data within the chorus emission frequency range. The highest frequency of emission above a threshold power spectral density was recorded. Most outliers represent noise and electrostatic emissions (not chorus). over longitude. The authors make the point that if the wave power at lower frequencies were higher than modeled, and if there was wave power at latitudes higher than 10 degrees, then the energy diffusion rates would be higher at higher energies leading to a reduced timescale for electron acceleration. Such estimates will require further detailed modeling, however, based on the improved observations. We have already commented on the inadequate sampling of the wave data versus local time. It will take future missions to determine the dependence of chorus intensity versus local time.

\section{Summary and conclusions}

Observations of the Galileo plasma wave instrument indicate that whistler mode chorus emissions with frequencyintegrated power levels of $10^{-8} \mathrm{~V}^{2} / \mathrm{m}^{2}$ or greater are observed commonly in the Jovian magnetosphere near the magnetic equator in the approximate radial range $6 R_{J}<r<10 R_{J}$. The survey includes over 30 orbits and nearly all local times but not all radial distances due to the spacecraft trajectory. The data seem to indicate that chorus emissions are more frequent and intense on the dayside, but there are far fewer orbital passes for the nightside in the range $6 R_{J}<r<12 R_{J}$, so no firm conclusions can be drawn. The orbit of Galileo is confined for most of the mission to near the Jovigraphic equator, but the tilt of the magnetic field permits coverage of a range of magnetic latitudes of $-10^{\circ}<\lambda_{\mathrm{mag}}<+10^{\circ}$.

The Jovian magnetosphere is rotationally-driven which provides the power for the interchange instability. As others have shown, warm plasma with a temperature anistropy from the outer magnetosphere provides a likely source of freeenergy for the growth of whistler-mode chorus in the inner magnetosphere $\left(6<r<10 R_{J}\right)$. The Jovian chorus emissions are seen below the electron cyclotron frequency, $f_{c e}$, in the approximate frequency range $400 \mathrm{~Hz}<f<8 \mathrm{kHz}$, with typical spectral density peaks near $10^{-10} \mathrm{~V}^{2} /\left(\mathrm{m}^{2}-\mathrm{Hz}\right)$ perhaps an order of magnitude lower than terrestrial chorus. The emission is relatively narrow-banded with maximum frequency and power a few degrees above the magnetic equator and decreasing rapidly at higher latitudes. While terrestrial chorus also displays both of these features, the decrease in frequency with latitude at Jupiter is larger than typically observed at Earth. A likely explanation for this is a large gradient in electron density at Jupiter away from the magnetic or centrifugal equator. We have examined the dependence of the frequency of chorus emission on magnetic latitude a little more carefully by plotting the upper frequency of the chorus emission as a function of magnetic latitude. To do this we normalized the frequency of the chorus electric field oscillations to $f_{c e}$, and used an algorithm to find the highest frequency of chorus emission above a threshold power spectral density. The threshold value was independently set for each chorus event. Within each frequency sweep the search was started at the 
highest frequency within the range of chorus emission. We eliminated a few of the chorus events because the emission was too bursty or had too many data gaps. A summary plot of the results (including over 14500 points) is included as Fig. 8. There are numerous outliers due to significant noise in the data and electrostatic emissions, but the salient feature of this plot is the general decrease in the upper frequency cutoff of the chorus emission with increasing latitude in both directions away from the equator, with a somewhat steeper slope in the direction of positive magnetic latitude. The centroid of the points also seems to be shifted about 1 degree to positive latitudes.

The global mapping of Jovian chorus in local time seen in Fig. 4 is interesting, showing regions of large power on the dayside of Jupiter and a lack of chorus at dawn for $r>10 R_{J}$, but the incomplete sampling of Galileo in local time and radius precludes any firm conclusions. We point out that since the Earth's magnetosphere is driven by the solar wind, while Jupiter is dominantly controlled by rotational dynamics, it would not be surprising to find a difference in global distribution of chorus power levels between the two planets.

Chorus emissions at Earth are now known to be a significant contributor to the acceleration of electrons in the Van Allen radiation belts. The similarities of Jovian chorus emissions to terrestrial observations are good reasons to assume that this emission may play a significant role in the stochastic acceleration of electrons in the radial range $6-10 R_{J}$, in the outer part of the Io torus where the density decreases and the ratio $f_{p} / f_{c e}$ falls to a level where the generation of chorus becomes efficient. As shown by Horne et al. (2008) these accelerated electrons may then be transported inward by radial diffusion where they are additionally accelerated to form the synchrotron radiation belt source. In the future the inclusion of effects such as the frequency dependence of chorus with latitude and in situ measurements of electrons will be important for advancing our knowledge of the effectiveness of chorus as well as other observed wave modes in accelerating Jovian electrons.

Acknowledgements. This work was supported by NASA contract to JPL 958779. We thank J. Hospodarsky for clerical support, and the reviewers for many helpful comments.

Topical Editor I. A. Daglis thanks N. P. Meredith and O. Santolik for their help in evaluating this paper.

\section{References}

Anderson, R. R. and Maeda, K.: VLF emissions associated with enhanced magnetospheric electrons, J. Geophys. Res., 82, 135146, 1977.

Bagenal, F.: Empirical model of the Io plasma torus: Voyager measurements, J. Geophys. Res., 99, 11 043-11 062, 1994.

Berge, G. L. and Gulkis, S.: Earth-based radio observations of Jupiter: Millimeter to meter wavelengths, in: Jupiter, edited by: Gehrels, T., The University of Arizona Press, Tucson, pp. 621$692,1976$.
Bolton, S. J., Thorne, R. M., Gurnett, D. A., Kurth, W. S., and Williams, D. J.: Enhanced whistler-mode emissions: Signatures of interchange motion in the Io torus, Geophys. Res. Lett., 24, 2123-2126, 1997.

Bolton, S. J., Levin, S. M., Gulkis, S. L., Klein, M. J., Sault, R. J., Bhattacharya, B, Thorne, R. M., Dulk, G. A., and Leblanc, Y.: Divine-Garrett model and Jovian synchrotron emission, Geophys. Res. Lett., 28(5), 907-910, 2001.

Bortnik, J., Thorne, R. M., and Meredith, N. P.: Modeling the propagation characteristics of chorus using CRRES suprathermal electron fluxes, J. Geophys. Res., 112, A08204, doi:1029/2006JA012237, 2007.

Bortnik, J., Thorne, R. M., and Meredith, N. P.: The unexpected origin of plasmaspheric hiss from discrete chorus emissions, Nature, 452, 62, doi:10.1038/nature06741, 2008.

Brice, N. and McDonough, T. R.: Jupiter's radiation belts, Icarus, 18, 206-219, 1973.

Carr, T. D., Desch, M. D., and Alexander, J. K.: Phenomenology of magnetospheric radio emissions, in: Physics of the Jovian Magnetosphere, edited by: Dessler, A. J., Cambridge University Press, Cambridge, pp. 226-284, 1983.

Gough, M. P., Christiansen, P. J., Martelli, G., and Gershuny, E. J.: Interaction of electrostatic waves with warm electrons at the geomagnetic equator, Nature, 279, 515-517, 1979.

Gurnett, D. A. and Scarf, F. L.: Plasma waves in the Jovian magnetosphere, in: Physics of the Jovian Magnetosphere, edited by: Dessler, A. J., Cambridge University Press, Cambridge, pp. 285316, 1983.

Gurnett, D. A. and Bhattacharjee, A.: Introduction to Plasma Physics, Cambridge University Press, Cambridge, UK, p. 379, 2005.

Gurnett, D. A., Kurth, W. S., Shaw, R. R., Roux, A., Gendrin, R., Kennel, C. F., Scarf, F. L., and Shawhan, S. D.: The Galileo plasma wave investigation, Space Sci. Rev., 60, 341-355, 1992.

Hill, T. W., Dessler, A. J., and Goertz, C. K.: Magnetospheric models, in: Physics of the Jovian Magnetosphere, edited by: Dessler, A. J., Cambridge University Press, Cambridge, pp. 353-394, 1983.

Horne, R. B. and Thorne, R. M.: Potential waves for relativistic electron scattering and stochastic acceleration during magnetic storms, Geophys. Res. Lett., 25, 3011-3014, 1998.

Horne, R. B. and Thorne, R. M.: Relativistic electron acceleration and precipitation during resonant interactions with whistler-mode chorus, Geophys. Res. Lett., 30(10), 1527, doi:10.1029/2003GL016973, 2003.

Horne, R. B., Glauert, S. A., and Thorne, R. M.: Resonant diffusion of radiation belt electrons by whistler-mode chorus, Geophys. Res. Lett., 30(9), 1493, doi:10.1029/2003GL016963, 2003.

Horne R. B., Thorne, R. M., Shprits, Y. Y., Meredith ,N. P., Glauert, S. A., Smith, A. J., Kanekal, S. G., Baker, D. N., Engebretson, M. J., Posch, J. L., Spasojevic, M., Inan, U. S., Pickett, J. S., and Decreau, P. M, E.: Wave acceleration of electrons in the Van Allen radiation belts, Nature, 437, 227-230, doi:10.1038/nature03939, 2005.

Horne, R. B., Thorne, R. M., Glauert, S. A., Menietti, J. D., Shprits, Y. Y., and Gurnett, D. A.: Gyro-resonant electron acceleration at Jupiter, Nature Phys., 4, 301-304, doi:10.1038/nphys897, 2008.

Hospodarsky, G. B., Averkamp, T. F., Kurth, W. S., Gurnett, D. A., Santolik, O., and Dougherty, M. K.: Observations of chorus at 
Saturn using the Cassini radio and plasma wave instrument, J. Geophys. Res., in press, 2008.

Katoh, Y. and Omura, Y.: A study of generation mechanism of VLF triggered emission by self-consistent particle code, J. Geophys. Res., 111, A12207, doi:10.1029/2006JA011704, 2006.

Katoh, Y. and Omura, Y.: Computer simulation of chorus wave generation in the Earth's inner magnetosphere, Geophys. Res. Lett., 34, L03102, doi:10.1029/2006GL028594, 2007.

Kurth, W. S., Craven, J. D., Frank, L. A., and Gurnett, D. A.: Intense electrostatic waves near the upper hybrid resonance frequency, J. Geophys. Res., 84, 4145-4164, 1979.

Lauben, D. S., Inan, U. S., Bell, T. F., Kirchner, D. L., Hospodarsky, G. B., and Pickett, J. S.: VLF chorus emissions observed by POLAR during the January 10, 1997, magnetic cloud, Geophys. Res. Lett., 25, 2995-2998, 1998.

LeDocq, M. J., Gurnett, D. A., and Hospodarsky, G. B.: Chorus source locations from VLF Poynting flux measurements with the Polar spacecraft, Geophys. Res. Lett., 25, 4063-4066, 1998.

Menietti, J. D., Santolik, O., Rymer, A. M., Hospodarsky, G. B., Persoon, A. M., Gurnett, D. A., Coates, A. J., and Young, D. T.: Analysis of plasma waves observed within local plasma injections seen in Saturn's magnetosphere, J. Geophys. Res., 113, A05213, doi:10.1029/2007JA012856, 2008.

Meredith, N. P., Horne, R. B., and Anderson, R. R.: Substorm dependence of chorus amplitudes: Implications for the acceleration of electrons to relativistic energies, J. Geophys. Res., 106, 13 165-13 178, 2001.

Meredith, N. P., Cain, M., Horne, R. B., Thorne, R. M., Summers, D., and Anderson, R. R.: Evidence for chorus-driven electron acceleration to relativistic energies from a survey of geomagnetically disturbed periods, J. Geophys. Res., 108(A6), 1248, doi:10.1029/2002JA009764, 2003a.

Meredith, N. P., Horne, R. B., Thorne, R. M., and Anderson, R. R., Favored regions for chorus-driven electron acceleration to relativistic energies in the Earth's outer radiation belt, Geophys. Res. Lett., 30(16), 1871, doi:10.1029/2003GL017698, 2003b.

Nunn, D., Omura, Y., Matsumoto, H., Nagano, I., and Yagitani, S.: The numerical simulation of VLF chorus and discrete emissions observed on the Geotail satellite using a Vlasov code, J. Geophys. Res., 102(A12), 27 083-27 097, 1997.
Omura Y., Nunn, D., Matsumoto, H., and Rycroft, M. J.: A review of observational, theoretical and numerical studies of VLF triggered emissions, J. Atmos. Terr. Phys., 53, 351-368, 1991.

Pontius Jr., D. H., Hill, T. W., and Rassbach, M. E.: Steady state plasma transport in a corotation-dominated magnetosphere, Geophys. Res. Lett., 13, 1097-1100, 1986.

Santolik, O., Gurnett, D. A., Pickett, J. S., Parrot, M., and Cornilleau-Wehrlin, N.: Spatio-temporal structure of storm-time chorus, J. Geophys. Res., 108(A7), 1278, doi:10.1029/2002JA009791, 2003.

Santolik, O., Gurnett, D. A., Pickett, J. S., Parrot, M., and Cornilleau-Wehrlin, N.: A microscopic and nanoscopic view of storm-time chorus on 31 March 2001, Geophys. Res. Lett., 31, L02801, doi:10.1029/2003GL018757, 2004.

Summers, D., Thorne, R., and Xiao, F.: Relativistic theory of waveparticle resonant diffusion with application to electron acceleration in the magnetosphere, J. Geophys. Res., 103(A9), $20487-$ $20500,1998$.

Summers, D., Ma, C., Meredith, N. P., Horne, R. B., Thorne, R. M., Heynderickx, D., and Anderson, R. R.: Model of the energization of the outer-zone electrons by whistler-mode chorus during the October 9, 1990, geomagnetic storm, Geophys. Res. Lett., 29(24), 2174, doi:10.1029/2002GL016039, 2002.

Thorne, R. M.: Microscopic plasma processes in the Jovian magnetosphere, in: Physics of the Jovian magnetosphere, edited by: Dessler, A. J., Cambridge University Press, Cambridge, pp. 454 488, 1983.

Thorne, R. M., Armstrong, T. P., Stone, S., Williams, D. J., McEntrie, R. W., Bolton, S. J., Gurnett, D. A., and Kivelson, M. G.: Galileo evidence for rapid interchange transport in the Io torus, Geophys. Res. Lett., 24, 2131-2134, 1997.

Trakhtengerts, V. Y.: A generation mechanism for chorus emission, Ann. Geophys., 17, 95-100, 1999, http://www.ann-geophys.net/17/95/1999/.

Tsurutani, B. T. and Smith, E. J.: Postmidnight chorus: A substorm phenomenon, J. Geophys. Res., 79, 118-127, 1974. 